\title{
BEST SIMULTANEOUS APPROXIMATION OF QUASI-CONTINUOUS FUNCTIONS BY MONOTONE FUNCTIONS
}

\author{
SALEM M. A. SAHAB
}

(Received 28 June 1989)

Communicated by E. N. Dancer

\begin{abstract}
Let denote the Banach space (under the sup norm) of quasi-continuous functions on the unit interval $[0,1]$. Let denote the closed convex cone comprised of monotone nondecreasing functions on $[0,1]$. For $f$ and $g$ in and $1<p<\infty$, let $h_{p}$ denote the best $L_{p}$ simultaneous approximant of $f$ and $g$ by elements of $c$. It is shown that $h_{p}$ converges uniformly as $p \rightarrow \infty$ to a best $L_{\infty}$-simultaneous approximant of $f$ and $g$ by elements of $\mathscr{L}$. However, this convergence is not true in general for any pair of bounded Lebesgue measurable functions. If $f$ and $g$ are continuous, then each $h_{p}$ is continuous; so is $\lim _{p \rightarrow \infty} h_{p}=h_{\infty}$. 1980 Mathematics subject classification (Amer. Math. Soc.) (1985 Revision): primary 41 A 28; secondary 41 A 30,41 A 65.
\end{abstract}

\section{Introduction}

Let $\Omega$ be the real unit interval $[0,1]$. Let $\mu$ be the Lebesgue measure on $\Omega$ and let $\mathscr{A}$ be the collection of all measurable subsets of $\Omega$. Let $L_{p}(\Omega, \mathscr{A}, \mu), 1<p<\infty$, be the well known $L_{p}$ Banach spaces and let $\mathscr{Q} \subseteq L_{p}(\Omega, \mathscr{A}, \mu)$ be the Banach space (under the sup norm) comprised of all quasi-continuous functions defined on $\Omega$, that is, functions having at most discontinuities of the first kind only. Let $\mathscr{C} \subseteq \mathscr{Q}$ be the subspace of continuous functions on $\Omega$, and let $\mathscr{H}$ be the closed convex cone in $\mathscr{Q}$ consisting of all monotone nondecreasing functions on $\Omega$. Let $f$ and $g$ be two bounded Lebesgue measurable functions on $\Omega$. It was shown in [3] that

(C) 1991 Australian Mathematical Society 0263-6115/91 \$A2.00+0.00 
if $f \notin \mathscr{M}$ or $g \notin \mathscr{M}$, then there exists a unique $h_{p} \in \mathscr{M}, p \in(1, \infty)$, such that

$$
\left[\left\|f-h_{p}\right\|_{p}^{p}+\left\|g-h_{p}\right\|_{p}^{p}\right]^{1 / p}=\inf _{h \in \mathscr{M}}\left[\|f-h\|_{p}^{p}+\|g-h\|_{p}^{p}\right]^{1 / p} .
$$

We call $h_{p}$ the best $L_{p}$-simultaneous approximant of $f$ and $g$ by elements of $\mathscr{M}$. Unless indicated otherwise $h_{p}$ will be referred to as the b.s.a. of $f$ and $g$. In general we say that $f$ and $g$ have the simultaneous Polya property if $h_{\infty}=\lim _{p \rightarrow \infty} h_{p}$ is well defined as a bounded Lebesgue measurable function on $\Omega$.

When $f=g$ in $(0.1)$, we have the usual $L_{p}$-approximation of a single function $f$ by elements of $\mathscr{M}$. If $f_{p}$ is its best $L_{p}$-approximant, then $\lim _{p \rightarrow \infty} f_{p}=f_{\infty}$ exists provided $f$ is quasi-continuous, that is, $f$ has the Polya property in this case (see [2]). In this paper, we try primarily to generalize the results discussed in [2]. As for now, there has been no similar work concerning the convergence of $h_{p}$ as $p \rightarrow \infty$.

We devote the next section to studying the case when both $f$ and $g$ are real-valued functions defined on a finite point set $X$. We state formulas for computing $h_{p}$ and $h_{\infty}$ in this case, and we establish the basic convergence results needed later.

In Section 2, we utilize the results of Section 1 to establish convergence results in the space of step functions defined on $\Omega$.

The fact that the step functions are dense in the space of quasi-continuous functions together with the results of Section 2, enable us to obtain the simultaneous Polya property. This is done in Section 3 where we establish as well the continuity of $h_{p}, p>1$, whenever $f$ ang $g$ are continuous.

In Section 4, we show by an example that the simultaneous Polya property does not hold in general for any pair of bounded Lebesgue measurable functions. In particular, we consider the case when $f$ is approximately continuous on $\Omega$.

Throughout this report we may assume (unless otherwise indicated) that either $f$ and/or $g$ does not belong to $\mathscr{M}$.

\section{Best $L_{p}$-simultaneous approximation on a finite set}

Let $X=\left\{x_{1}, x_{2}, \ldots, x_{n}\right\}$ be a finite subset of $\mathscr{R}$ with $x_{1}<x_{2}<$ $\cdots<x_{n}$. Let $B=B(X)$ be the linear space of real functions on $X$ and $\mathscr{M}=\mathscr{M}(X)$ the closed convex cone of monotone nondecreasing functions in $B$, that is, functions $h$ satisfying $h(x) \leq h(y)$ whenever $x, y \in X$ and 
$x \leq y$. For each $p \in[1, \infty)$, define a weighted $L_{p}$-norm ${ }_{w}\|\cdot\|_{p}$ by

$$
{ }_{w}\|f\|_{p}=\left(\sum_{i=1}^{n} w_{i}\left|f_{i}\right|^{p}\right)^{1 / p}
$$

where $f=\left\{f_{i}\right\}_{i=1}^{n}=\left\{f\left(x_{i}\right)\right\}_{i=1}^{n} \in B$, and $w=\left\{w_{i}\right\}_{i=1}^{n}>0$ is a weight function satisfying $\sum_{i=1}^{n} w_{i}=1$.

Let $f=\left\{f_{i}\right\}_{i=1}^{n}$ and $g=\left\{g_{i}\right\}_{i=1}^{n}$ in $B$ be fixed. For each $p \in[1, \infty)$, a function $h_{p}=\left\{h_{p, i}\right\}_{i=1}^{n} \in \mathscr{K}$ is called a best weighted $L_{p}$-simultaneous approximant of $f$ and $g$ if

$$
\begin{aligned}
\left({ }_{w} \| f\right. & \left.-h_{p}\left\|_{p}^{p}+{ }_{w}\right\| g-h_{p} \|_{p}^{p}\right)^{1 / p} \\
& =\inf \left\{\left({ }_{w}\|f-h\|_{p}^{p}+{ }_{w}\|g-h\|_{p}^{p}\right)^{1 / p}: h \in \mathscr{M}\right\},
\end{aligned}
$$

or,

$$
\begin{gathered}
{\left[\sum_{i=1}^{n} w_{i}\left(\left|f_{i}-h_{p, i}\right|^{p}+\left|g_{i}-h_{p, i}\right|^{p}\right)\right]^{1 / p}} \\
\leq\left[\sum_{i=1}^{n} w_{i}\left(\left|f_{i}-h_{i}\right|^{p}+\left|g_{i}-h_{i}\right|^{p}\right)\right]^{1 / p},
\end{gathered}
$$

for all $h=\left\{h_{i}: i=1, \ldots, n\right\} \in \mathscr{M}$.

It was shown in [3] that $h_{p}$ is unique (up to equivalence) when $p \in$ $(1, \infty)$.

To this end we shall discuss briefly the computation of the values of $h_{p}$ explicitly, we start with the following definitions.

Definition. A subset $L \subseteq X$ is said to be a lower set if $x_{i} \in L$ and $x_{j} \in X, x_{j} \leq x_{i}$, imply that $x_{j} \in L$. Similarly, $U \subseteq X$ is an upper set if $x_{i} \in U$ and $x_{j} \in X, x_{j} \geq x_{i}$, imply that $x_{j} \in X$. For simplicity we will write $i \in Y \subseteq X$ instead of $x_{i} \in Y$.

Fix $p \in(1, \infty)$. If $L \cap U$ is nonempty, define $\mu_{p}(L \cap U)$ to be the unique real number minimizing

$$
\left\{\sum_{j} w_{j}\left[\left|f_{j}-u\right|^{p}+\left|g_{j}-u\right|^{p}\right]: j \in L \cap U\right\} .
$$

Let $h_{p}=\left\{h_{p, i}: i=1,2, \ldots, n\right\}$ be the function defined on $X$ by

$$
\begin{aligned}
h_{p, i} & =\max _{\{U: i \in U\}\{L: i \in L\}} \min _{p} \mu_{p}(L \cap U), \\
& =\min _{\{L: i \in L\} U U: i \in U\}} \max _{p}(L \cap U) .
\end{aligned}
$$


It is shown in [8, pages 21-38], that $h_{p}$ is the unique solution satisfying (1.2). Before we proceed we remind the reader of the sup norm defined by

$$
\|f\|_{\infty}=\max \left\{\left|f_{i}\right|: i=1, \ldots, n\right\} \text {. }
$$

Definition. Let $a=\min \left\{-\|f\|_{\infty},-\|g\|_{\infty}\right\}$ and $b=\max \left\{\|f\|_{\infty},\|g\|_{\infty}\right\}$. For fixed $f=\left\{f_{i}\right\}_{i=1}^{n}$ and $g=\left\{g_{i}\right\}_{i=1}^{n}$, we define functions $\tau_{p}:[a, b]^{n} \rightarrow \mathscr{R}$ and $\kappa_{p}:[a, b] \rightarrow \mathscr{R}$ for $1 \leq p \leq \infty$ by

$$
\tau_{p}(\bar{u})=\sum_{i=1}^{n} w_{p, i}\left(\left|f_{i}-u_{i}\right|^{p}+\left|g_{i}-u_{i}\right|^{p}\right), \quad p<\infty,
$$

$$
\tau_{\infty}(\bar{u})=\max _{1 \leq i \leq n}\left(\left|f_{i}-u_{i}\right|,\left|g_{i}-u_{i}\right|\right)
$$

$$
\kappa_{p}(u)=\sum_{i=1}^{n} w_{p, i}\left(\left|f_{i}-u\right|^{p}+\left|g_{i}-u\right|^{p}\right),
$$

$$
\kappa_{\infty}(u)=\max _{1 \leq i \leq n}\left(\left|f_{i}-u\right|,\left|g_{i}-u\right|\right),
$$

where $\bar{u}=\left(u_{1}, \ldots, u_{n}\right) \in[a, b]^{n}$ and $u \in[a, b]$.

LEMMA 1. Suppose $w_{p}=\left\{w_{p, i}\right\}_{i=1}^{n}>0$ with $\sum_{i=1}^{n} w_{p, i}=1$, and

$$
\liminf _{p \rightarrow \infty} w_{p, i}>0 \text {. }
$$

Then

$$
\lim _{p \rightarrow \infty}\left(\tau_{p}(\bar{u})\right)^{1 / p}=\tau_{\infty}(\bar{u})
$$

and

$$
\lim _{p \rightarrow \infty}\left(\kappa_{p}(u)\right)^{1 / p}=\kappa_{\infty}(u),
$$

and the convergence is uniform on the compact sets $[a, b]^{n}$ and $[a, b]$ respectively.

Proof. From (1.8) we conclude that there exist real numbers $\delta_{1}, \delta_{2} \in$ $(0,1]$ and $p_{0} \geq 1$ such that $w_{p, i} \in\left[\delta_{1}, \delta_{2}\right]$ for all $i$ and all $p \geq p_{0}$. Hence

$$
\begin{aligned}
\tau_{p}(\bar{u}) & =\sum_{i=1}^{n} w_{p, i}\left(\left|f_{i}-u_{i}\right|^{p}+\left|g_{i}-u_{i}\right|^{p}\right), \\
& \leq \sum_{i=1}^{n} \delta_{2}\left[\left(\tau_{\infty}(\bar{u})\right)^{p}+\left(\tau_{\infty}(\bar{u})\right)^{p}\right], \\
& =\sum_{i=1}^{n} 2 \delta_{2}\left(\tau_{\infty}(\bar{u})\right)^{p}=2 n \delta_{2}\left(\tau_{\infty}(\bar{u})\right)^{p}
\end{aligned}
$$


for all $p \geq p_{0}$. In other words,

$$
(\tau(\bar{u}))^{1 / p}-\tau_{\infty}(\bar{u}) \leq\left[\left(2 n \delta_{2}\right)^{1 / p}-1\right] \tau_{\infty}(\bar{u}) .
$$

Since $X$ is finite, we may assume without loss of generality the existence of an integer $k, 1 \leq k \leq n$, such that

$$
\tau_{\infty}(\bar{u})=\left|f_{k}-u_{k}\right|
$$

Hence,

$$
\tau_{p}(\bar{u}) \geq w_{p, k}\left[\left|f_{k}-u_{k}\right|\right]^{p} \geq \delta_{1}\left(\tau_{\infty}(\bar{u})\right)^{p},
$$

which implies that

$$
\left(\delta_{1}^{1 / p}-1\right) \tau_{\infty}(\bar{u}) \leq\left(\tau_{p}(\bar{u})\right)^{1 / p}-\tau_{\infty}(\bar{u})
$$

Combining (1.11) and (1.12) it follows that

$$
\left|\tau_{p}(\bar{u})^{1 / p}-\tau_{\infty}(\bar{u})\right| \leq \max \left\{\left|\left(2 n \delta_{2}\right)^{1 / p}-1\right|,\left|\delta_{1}^{1 / p}-1\right|\right\} \tau_{\infty}(\bar{u}) .
$$

Now $\tau_{\infty}(\bar{u})$ is continuous in $\bar{u}$, so it is bounded on the compact domain $[a, b]^{n}$. Hence the uniform convergence of $\left(\tau_{p}(\bar{u})\right)^{1 / p}$ to $\tau_{\infty}(\bar{u})$ follows. This establishes (1.9). To obtain $(1.10)$ take $\bar{u}=(u, \ldots, u)$.

REMARK. In the above lemma, we may waive the requirement that

$$
\sum_{i=1}^{n} w_{p, i}=1 \text {, }
$$

if we require instead that

$$
0<\liminf _{p \rightarrow \infty} w_{p, i} \leq \limsup _{p \rightarrow \infty} w_{p, i}<\infty
$$

The proof is essentially the same. See [7, Theorem 1] for a similar argument.

LEMma 2. For $1<p \leq \infty, \kappa_{p}(u)$ has a unique minimizer $u_{p}$. Moreover,

$$
\lim _{p \rightarrow \infty} u_{p}=u_{\infty}
$$

and $u_{\infty}$ is the minimizer of $\kappa_{\infty}(u)$.

Proof. The proof of [7, Lemma 2] can be modified to obtain the desired result. 
Theorem 3. Assume that $\omega_{p}=\left\{\omega_{p, i}\right\}_{i=1}^{n}>0$ satisfies (1.8) and

$$
\sum_{i=1}^{n} \omega_{p, i}=1 \text { for all } p, 1<p<\infty .
$$

Then the solution $h_{p}=\left\{h_{p, i}: i=1, \ldots, n\right\}$ given by (1.3) and satisfying (1.2) converges as $p \rightarrow \infty$ to a solution $h_{\infty}=\left\{h_{\infty, i}: i=1, \ldots, n\right\}$ satisfying

$$
\begin{aligned}
& \max \left(\left\|f-h_{\infty}\right\|_{\infty},\left\|g-h_{\infty}\right\|_{\infty}\right) \\
& \quad=\inf \left\{\max \left(\|f-h\|_{\infty},\|g-h\|_{\infty}\right): h \in \mathscr{M}\right\},
\end{aligned}
$$

or,

$$
\max _{1 \leq i \leq n}\left(\left|f_{i}-h_{\infty, i}\right|,\left|g-h_{\infty, i}\right|\right) \leq \max _{1 \leq i \leq n}\left(\left|f_{i}-h_{i}\right|,\left|g_{i}-h_{i}\right|\right), \quad h=\left\{h_{i}\right\}_{i=1}^{n} \in \mathscr{M}
$$

Moreover

$$
\begin{aligned}
h_{\infty, i} & =\lim _{p \rightarrow \infty} h_{p, i}=\max _{\{U: i \in U\}\{L: i \in L\}} \min _{\infty}(L \cap U) \\
& =\min _{\{L: i \in L\}\{U: i \in U\}} \mu_{\infty}(L \cap U),
\end{aligned}
$$

for every $i$, where $L$ and $U$ are lower and upper sets respectively and $\mu_{\infty}(L \cap U)$ is the unique real number minimizing

$$
\max _{j \in L \cap U}\left(\left|f_{j}-u\right|,\left|g_{j}-u\right|\right)
$$

for all real $u$.

Proof. Putting $L \cap U$ instead of $X$ in Lemma 2 above we conclude that

$$
\lim _{p \rightarrow \infty} \mu_{p}(L \cap U)=\mu_{\infty}(L \cap U)
$$

exists and $\mu_{\infty}(L \cap U)$ is the minimizer of $\max \left\{\left|f_{j}-u\right|,\left|g_{j}-u\right|: j \in L \cap U\right\}$. Since $X$ is finite, the number of lower and upper sets is finite, so (1.3) implies that the limit of $h_{p, i}$ exists as $p \rightarrow \infty$ for all $i$, and therefore (1.14) holds and it has a real value, say $h_{\infty, i}, i=1, \ldots, n$.

It remains to show that (1.13) holds, that is, $h_{\infty}$ is indeed a best $L_{\infty}$ simultaneous approximant of $f$ and $g$. Since $\left\{h_{p, i}\right\}_{i=1}^{n} \in \mathscr{M}$ for every $p<\infty,\left\{h_{\infty, i}\right\}_{i=1}^{n} \in \mathscr{M}$. Clearly

$$
\min \left(f_{i}, g_{i}\right) \leq h_{p, i} \leq \max \left(f_{i}, g_{i}\right),
$$

for all $p$ and $i$. By definition of $\left\{h_{p, i}\right\}_{i=1}^{n}$, we have $\left(\tau_{p}\left(h_{p}\right)\right)^{1 / p} \leq\left(\tau_{p}(\bar{u})\right)^{1 / p}$ 
for all $\bar{u} \in \mathscr{R}^{n}$. Now, let $p \rightarrow \infty$ to conclude from (1.9) that $\tau_{\infty}\left(h_{\infty}\right) \leq$ $\tau_{\infty}(\bar{u})$ for all $\bar{u} \in \mathscr{R}^{n}$. Hence $h_{\infty}$ satisfies (1.13). This completes the proof.

\section{B.s.a. to step functions}

Definition. Let $\pi$ be a finite partition $\Omega$ with points $\left\{t_{i}: i=0,1, \ldots\right.$, $n$ \} such that $0=t_{0}<t_{1}<\cdots<t_{n}=1$. Let $I_{E}$ denote the indicator function of a subset $E$ of $\Omega$. Let $S_{\pi}$ be the linear space comprised of all step functions of the form

$$
f=\sum_{i=1}^{n} f_{i} I_{\left(t_{i}-1, t_{i}\right]},
$$

where $f_{i} \in \mathscr{R}$ for every $i$, and for $i=1$, we include the point $t_{0}=0$.

LEMMA 4. Let $f$ and $g$ be in $S_{\pi}$ and let $h_{p}, 1<p<\infty$, be the b.s.a. of $f$ and $g$ by elements of $\mathscr{K}$. Then $h_{p} \in S_{\pi}$.

Proof. Suppose $h_{p}$ is not constant on some sub-interval $\left(t_{j}-1, t_{j}\right]$. Let

$$
l=\operatorname{essinf}\left\{h_{p}(t): t_{j-1}<t \leq t_{j}\right\},
$$

and

$$
u=\operatorname{essup}\left\{h_{p}(t): t_{j-1}<t \leq t_{j}\right\}
$$

Clearly $l<u$. Choose $\zeta \in[l, u]$ such that

$$
\left|f_{j}-\zeta\right|^{p}+\left|g_{j}-\zeta\right|^{p}=\inf \left\{\left|f_{j}-r\right|^{p}+\left|g_{j}-r\right|^{p}: r \in[l, u]\right\}
$$

where $f_{j}$ and $g_{j}$ are respectively the values of $f$ and $g$ on $\left(t_{j-1}, t_{j}\right]$. Now, let $h_{p}^{*}$ be the element of $\mathscr{K}$ defined by

$$
h_{p}^{*}(t)= \begin{cases}\zeta, & t_{j-1}<t \leq t_{j} \\ h_{p}(t), & \text { otherwise }\end{cases}
$$


Then $h_{p}^{*}$ is a better b.s.a. of $f$ and $g$ since

$$
\begin{aligned}
\left\|f-h_{p}^{*}\right\|_{p}^{p}+\left\|g-h_{p}^{*}\right\|_{p}^{p}= & \sum_{\substack{i=1 \\
i \neq j}}^{n} \int_{t_{i-1}}^{t_{i}}\left(\left|f_{i}-h_{p}(t)\right|^{p}+\left|g_{i}-h_{p}(t)\right|^{p}\right) d t \\
& +\int_{t_{j-1}}^{t_{j}}\left(\left|f_{j}-\zeta\right|^{p}+\left|g_{j}-\zeta\right|^{p}\right) d t \\
& <\sum_{\substack{i=1 \\
i \neq j}}^{n} \int_{t_{i-1}}^{t_{i}}\left(\left|f_{i}-h_{p}(t)\right|^{p}+\left|g_{i}-h_{p}(t)\right|^{p}\right) d t \\
& +\int_{t_{j-1}}^{t_{j}}\left(\left|f_{j}-h_{p}(t)\right|^{p}+\left|g_{j}-h_{p}(t)\right|^{p}\right) d t,
\end{aligned}
$$

or,

$$
\left\|f-h_{p}^{*}\right\|_{p}^{p}+\left\|g-h_{p}^{*}\right\|_{p}^{p}<\left\|f-h_{p}\right\|_{p}^{p}+\left\|g-h_{p}\right\|_{p}^{p},
$$

a contradiction. Therefore $h_{p}$ must be constant on $\left(t_{j}-1, t_{j}\right]$, or $h_{p} \in S_{\pi}$.

LEMMA 5. Let $p \in(1, \infty)$ be fixed. Let $f_{1}, f_{2}, g_{1}$ and $g_{2}$ be elements of $S_{\pi}$. Let $h_{1}$ and $h_{2}$ be the b.s.a. of $f_{1}, g_{1}$ and $f_{2}, g_{2}$ respectively. If $f_{1} \leq f_{2}$ and $g_{1} \leq g_{2}$, then $h_{1} \leq h_{2}$.

Proof. It was shown in [5, Lemma 2] that for $1<p<\infty$ and for all real numbers $a, b, c, d$ with $a \geq c$,

$$
|a-b|^{p}+|c-d|^{p} \geq|a-\max (b, d)|^{p}+|c-\min (b, d)|^{p} .
$$

Define functions $T_{1}$ and $T_{2}$ by $T_{1}(x)=\min \left(h_{1}(x), h_{2}(x)\right)$ and $T_{2}(x)=$ $\max \left(h_{1}(x), h_{2}(x)\right)$. Applying $(2.2)$ at every $x \in[0,1]$ with $a=f_{2}(x)$, $b=h_{2}(x), c=f_{1}(x)$ and $d=h_{1}(x)$, we obtain

$$
\left|f_{2}(x)-h_{2}(x)\right|^{p}+\left|f_{1}(x)-h_{1}(x)\right|^{p} \geq\left|f_{2}(x)-T_{2}(x)\right|^{p}+\left|f_{1}(x)-T_{1}(x)\right|^{p},
$$

and hence by integrating over $[0,1]$ we get

$$
\left\|f_{2}-h_{2}\right\|_{p}^{p}+\left\|f_{1}-h_{1}\right\|_{p}^{p} \geq\left\|f_{2}-T_{2}\right\|_{p}^{p}+\left\|f_{1}-T_{1}\right\|_{p}^{p} .
$$

Similarly, we obtain

$$
\left\|g_{2}-h_{2}\right\|_{p}^{p}+\left\|g_{1}-h_{1}\right\|_{p}^{p} \geq\left\|g_{2}-T_{2}\right\|_{p}^{p}+\left\|g_{1}-T_{1}\right\|_{p}^{p} .
$$

Adding (2.3) to (2.4), we conclude that either

$$
\left\|f_{2}-h_{2}\right\|_{p}^{p}+\left\|g_{2}-h_{2}\right\|_{p}^{p} \geq\left\|f_{2}-T_{2}\right\|_{p}^{p}+\left\|g_{2}-T_{2}\right\|_{p}^{p},
$$

or

$$
\left\|f_{1}-h_{1}\right\|_{p}^{p}+\left\|g_{1}-h_{1}\right\|_{p}^{p} \geq\left\|f_{1}-T_{1}\right\|_{p}^{p}+\left\|_{p}^{p}+\right\| g_{1}-T_{1} \|_{p}^{p}
$$


or both of them. If $(2.5)$ holds, then by definition of $h_{2}$ we must have

$$
h_{2}=T_{2}=\max \left(h_{1}, h_{2}\right) \geq h_{1} \text {. }
$$

If (2.6) holds, then we end up with $h_{1}=T_{1} \leq h_{2}$. This completes the proof.

LeMMA 6. Let $f$ and $g$ be elements of $S_{\pi}$, and let $h_{p}$ be their b.s.a. Then $h_{p}+c$ is the b.s.a. of $f+c$ and $g+c$ where $c \in \mathscr{R}$.

Proof. This is clear, since $h_{p}+c \in \mathscr{M}$ for all $c$.

REMARK. The last two lemmas are true in general for all bounded Lebesgue measurable functions on $[0,1]$. The proofs are essentially the same.

THEOREM 7. Let $f$ and $g$ be elements of $S_{\pi}$ given by

$$
f=\sum_{i=1}^{n} f_{i} I_{\left(t_{i-1}, t_{i}\right]},
$$

and

$$
g=\sum_{i=1}^{n} g_{i} I_{\left(t_{i-1}, t_{i}\right)}
$$

For every $p, 1<p<\infty$, let $\omega_{p}=\left\{\omega_{p, i}\right\}_{i=1}^{n}$ be defined by $\omega_{p, i}=t_{i}-t_{i-1}$ for all $i$, and let $h_{p}=\left\{h_{p, i}\right\}_{i=1}^{n}$ be given by (1.3). Then, the b.s.a. of $f$ and $g$ is given by

$$
h_{p}^{*}=\sum_{i=1}^{n} h_{p, i} I_{\left(t_{i-1}, t_{i}\right]}
$$

Proof. By Lemma 4, we know that $h_{p}^{*} \in S_{\pi}$. Let $X=\left\{x_{1}, \ldots, x_{n}\right\}$, where $x_{i}=\left(t_{i}+t_{i-1}\right) / 2, i=1, \ldots, n$. Consider $\left\{f_{i}: i=1, \ldots, n\right\}$ and $\left\{g_{i}: i=1, \ldots, n\right\}$ as two finite real valued functions defined on $X$ and let $\left\{h_{i}: i=1, \ldots, n\right\}, h_{i} \leq h_{j}$ for all $i<j$, be a monotone nondecreasing function on $X$. Then by substituting the values of $\omega_{p, i}$ in equation $\left(1.2^{\prime}\right)$ we obtain

$$
\begin{aligned}
& {\left[\sum_{i=1}^{n}\left(t_{i}-t_{i-1}\right)\left(\left|f_{i}-h_{p, i}\right|^{p}+\left|g_{i}-h_{p, i}\right|^{p}\right)\right]^{1 / p}} \\
& \leq\left[\sum_{i=1}^{n}\left(t_{i}-t_{i-1}\right)\left(\left|f_{i}-h_{i}\right|^{p}+\left|g_{i}-h_{i}\right|^{p}\right)\right]^{1 / p}
\end{aligned}
$$


or,

$$
\begin{gathered}
{\left[\sum_{i=1}^{n} \int_{t_{i-1}}^{t_{i}}\left(\left|f_{i}-h_{p, i}\right|^{p}+\left|g_{i}-h_{p, i}\right|^{p}\right)\right]^{1 / p}} \\
\leq\left[\sum_{i=1}^{n} \int_{t_{i-1}}^{t_{i}}\left(\left|f_{i}-h_{i}\right|^{p}+\left|g_{i}-h_{i}\right|^{p}\right)\right]^{1 / p},
\end{gathered}
$$

which implies that

$$
\left\|f-h_{p}^{*}\right\|_{p}^{p}+\left\|g-h_{p}^{*}\right\|_{p}^{p} \leq\|f-h\|_{p}^{p}+\|g-h\|_{p}^{p},
$$

for any monotone nondecreasing function $h=\sum_{i=1}^{n} h_{i} I_{\left(t_{i-1}, t_{i}\right]}$ belonging to $S_{\pi}$.

REMARK. Using Theorem 7, we are able to compute $h_{p}, p \in(1, \infty)$, when $f$ and $g$ are in $S_{\pi}$ by applying (1.3). To compute $h_{\infty}$, we may use (1.14) and the following theorem.

THEOREM 8. Let $f, g$ and $h_{p}^{*}$ be as given above. Then $h_{p}^{*}$ converges as $p \rightarrow \infty$ to the monotone nondecreasing function $h_{\infty}^{*} \in S_{\pi}$ given by

$$
h_{\infty}^{*}=\sum_{i=1}^{n} h_{\infty, i} I_{\left(t_{i-1}, t_{i}\right]},
$$

where $h_{\infty, i}=\lim _{p \rightarrow \infty} h_{p, i}$ is given by (1.14). Furthermore, $h_{\infty}^{*}$ is a best $L_{\infty}$-simultaneous approximant of $f$ and $g$ by elements of $\mathscr{M}$.

Proof. Let $X$ and $\omega_{p}$ be as defined above. Then Theorem 3 implies the first part of the theorem. It also implies that $h_{\infty}^{*}$ is a best $L_{\infty}$-simultaneous approximant of $f$ and $g$ by monontone nondecreasing functions in $S_{\pi}$.

Let $h$ be any monotone nondecreasing function on [0,1]. We show that there is a monotone nondecreasing function $h^{*} \in S_{\pi}$ such that

$$
\max \left(\left\|f-h^{*}\right\|_{\infty},\left\|g-h^{*}\right\|_{\infty}\right) \leq \max \left(\|f-h\|_{\infty},\|g-h\|_{\infty}\right) .
$$

Indeed, for $i=1,2, \ldots, n$, let

$$
h_{i}^{*}=(1 / 2)[\operatorname{essup}(h(x))+\operatorname{essinf}(h(x))], \quad t_{i-1}<x \leq t_{i} .
$$

Then clearly

$$
\left|f_{i}-h_{i}^{*}\right| \leq \operatorname{essup}\left|f_{i}-h(x)\right|, \quad t_{i-1}<x \leq t_{i},
$$

and

$$
\left|g_{i}-h_{i}^{*}\right| \leq \operatorname{essup}\left|g_{i}-h(x)\right|, \quad t_{i-1}<x \leq t_{i},
$$


for all $i$. Hence,

$$
\max \left(\left|f_{i}-h_{i}^{*}\right|,\left|g_{i}-h_{i}^{*}\right|\right) \leq \max \left(\operatorname{essup}\left|f_{i}-h(x)\right|, \text { essup }\left|g_{i}-h(x)\right|\right)
$$

for all $i$. Now define $h^{*} \in S_{\pi}$ by

$$
h^{*}=\sum_{i=1}^{n} h_{i}^{*} I_{\left(t_{i-1}, t_{i}\right.} .
$$

Then surely we conclude from the last inequality that

$$
\max \left(\left\|f-h^{*}\right\|_{\infty},\left\|g-h^{*}\right\|_{\infty}\right) \leq\left(\|f-h\|_{\infty},\|f-g\|_{\infty}\right) .
$$

This completes the proof.

REMARK. We have seen that for $f, g \in S_{\pi}$, we also have $h_{p}, h_{\infty} \in S_{\pi}$. Let us define $S$ to be the collection of all $S_{\pi}$, that is,

$$
S=\left\{\bigcup_{\pi} S_{\pi}: \pi \text { is any finite partition of }[0,1]\right\} .
$$

If $f$ and $g$ are elements of a particular $S_{\pi}$, then we use the notation $f_{\pi}, g_{\pi}$. Let $h_{\pi, p}$ denote the best $L_{p}$-simultaneous approximant of $f_{\pi}$ and $g_{\pi}$ and let $h_{\pi, \infty}=\lim _{p \rightarrow \infty} h_{\pi, p}$ denote the limit of $h_{\pi, p}$ as $p$ increases to infinity.

\section{B.s.a. of quasi-continuous and continuous functions}

Definition. A function $f:[0,1] \rightarrow \mathscr{R}$ is said to be quasi-continuous if it has at most discontinuities of the first kind only. Let $\mathscr{Q}$ be the Banach space (sup norm) consisting of all quasi-continuous functions on $\Omega$.

Let $\mathscr{Q}^{*}$ denote the space of all elements of such that $f(0)=f\left(0^{+}\right)$ and $f(x)=f\left(x^{-}\right), 0<x \leq 1$. Then there is a linear isometry between $\mathscr{Q}^{*}$ and $\mathscr{Q}^{2}$, so that we may work with elements of $\mathscr{Q}^{*}$ only. For simplicity we denote $\mathscr{Q}^{*}$ by $\mathscr{Q}$. Hence $S$ as defined in Section 1 is a proper subset of $\mathscr{Q}$. We also regard $\mathscr{M}$ as a proper subset of $\mathscr{Q}$, so in fact $\mathscr{M}=\mathscr{M} \cap \mathscr{Q}^{*}$.

Definition. Let $f$ be a bounded Lebesgue measurable function on $[0,1]$, and let $\pi$ be a partition of $[0,1]$. Then $\bar{f}_{\pi}$ in $S$ is defined by

$$
\bar{f}_{\pi}(x)=\sup \left\{f(y): y \in\left(t_{i-1}, t_{i}\right]\right\}, \quad x \in\left(t_{i-1}, t_{i}\right], \quad i \geq 1 .
$$

We define $\underline{f}_{\pi}$ similarly by replacing sup by inf.

A bounded function $f$ is in 2 if and only if, for any $\varepsilon>0$, there exists a partition $\pi$ of $[0,1]$ such that $0 \leq \bar{f}_{\pi}-\underline{f}_{\pi}<\varepsilon$. Thus, $\lim _{\pi} \bar{f}_{\pi}=\lim _{\pi} \underline{f}_{\pi}=$ $f$. Moreover, if $\pi^{\prime}$ is a refinement partition of $\pi$, then we have

$$
\underline{f}_{\pi} \leq \underline{f}_{\pi^{\prime}} \leq \bar{f}_{\pi^{\prime}} \leq \bar{f}_{\pi} \text {. }
$$


For more details see [2]. Thus, when $f, g \in \mathscr{Q}$, we are able to get as close as we like to $f$ and $g$. Of course, we count here on the fact that the projection map $(f, g) \mapsto h_{p}$ is continuous.

Lemma 9. Let $f, g \in \mathscr{Q}$, and let $\varepsilon>0$ be given. Let $\pi$ be a partition such that $0 \leq \bar{f}_{\pi}-\underline{f}_{\pi}<\varepsilon$ and $0 \leq \bar{g}_{\pi}-\underline{g}_{\pi}<\varepsilon$. Then there exists $a$ refinement $\pi^{\prime}$ of $\pi$ such that

$$
\underline{h}_{\pi, p} \leq \underline{h}_{\pi^{\prime}, p} \leq \bar{h}_{\pi^{\prime}, p} \leq \bar{h}_{\pi, p} \leq \underline{h}_{\pi, p}+\varepsilon,
$$

and

$$
\underline{h}_{\pi, \infty} \leq \underline{h}_{\pi^{\prime}, \infty} \leq \bar{h}_{\pi^{\prime}, \infty} \leq \bar{h}_{\pi, \infty} \leq \underline{h}_{\pi, \infty}+\varepsilon,
$$

where these $h$ 's are as defined earlier in the last remark at the end of Section 2 .

Proof. The discussion preceding the statement of the lemma implies that (3.2) holds for both $f$ and $g$ with the addition of $\bar{f}_{\pi}<\underline{f}_{\pi}+\varepsilon$, and $\bar{g}_{\pi}<\underline{g}_{\pi}+\varepsilon$. Apply Lemmas 8 and 9 to conclude (3.3). Letting $p \rightarrow \infty$ gives us (3.4).

The proof of the next theorem can be obtained following the same line of proof as [2, Theorems 4 and 5], respectively, with the proper changes in the notations used.

TheOREM 10. Let $f, g \in \mathscr{Q}$, and let $h_{p}, p \in(1, \infty)$, be their b.s.a.. Then

$$
\begin{gathered}
\lim _{\pi} \bar{h}_{\pi, p}=\lim _{\pi} \underline{h}_{\pi, p}=h_{p}, \\
\lim _{\pi} \bar{h}_{\pi, \infty}=\lim _{\pi} \underline{h}_{\pi, \infty}=h_{\infty}=\lim _{p \rightarrow \infty} h_{p} .
\end{gathered}
$$

The convergence being uniform in both cases.

Remark. (a) Let $f, g, h_{p}, h_{\infty}$ be as defined above. Let $h_{p}^{c}$ be the best $L_{p}$-simultaneous approximant of $f+c$ and $g+c$. Then, $\lim _{p \rightarrow \infty} h_{p}^{c}=$ $h_{\infty}+c$, where $c \in \mathscr{R}$.

(b) Let $f_{1} \leq f_{2}, g_{1} \leq g_{2}$, and let $h_{1, p}, h_{2, p}$ be the b.s.a. of $f_{1}, g_{1}$ and $f_{2}, g_{2}$ respectively, $1<p<\infty$. If $h_{k, \infty}=\lim _{p \rightarrow \infty} h_{k, p}, k=1,2$, then $h_{1, \infty} \leq h_{2, \infty}$.

Our next and final result in this section is a generalization of [2, Theorem 6]. 
THEOREM 11. Suppose $f$ and $g$ are continuous. Then $h_{p}$ is continuous, and so is $h_{\infty}$.

Proof. The second part of the conclusion is immediate once the first part is established. So let $x$ be an arbitrary but fixed point in $(0,1)$, and let $\varepsilon>0$ be given. Then

$$
\begin{aligned}
\left|h_{p}(x)-h_{p}(y)\right| \leq & \left|h_{p}(x)-\bar{h}_{\pi, p}(x)\right|+\left|\bar{h}_{\pi, p}(x)-\bar{h}_{\pi, p}(y)\right| \\
& +\left|\bar{h}_{\pi, p}(y)-h_{p}(y)\right| .
\end{aligned}
$$

Since

$$
h_{p}(t)=\lim _{\pi} \bar{h}_{\pi, p}(t)
$$

for all $t \in \Omega$, we may choose a partition $\pi=\left\{t_{i}: i=0,1, \ldots, n\right\}$ such that

(i) each of the first and third term on the right-hand side of (3.7) is less than $\varepsilon / 3$.

(ii) $f_{\pi}$ and $g_{\pi}$ can be written as

$$
\bar{f}_{\pi}=\sum_{i=1}^{n} \bar{a}_{i} I_{\left(t_{i-1}, t_{i}\right]},
$$

and

$$
\bar{g}_{\pi}=\sum_{i=1}^{n} \bar{b}_{i} I_{\left(t_{i-1}, t_{i}\right]},
$$

with

$$
\left|\bar{a}_{i}-\bar{a}_{i-1}\right|<\varepsilon / 9 \text {, }
$$

and

$$
\left|\bar{b}_{i}-\bar{b}_{i-1}\right|<\varepsilon / 9
$$

for all $i=2,3, \ldots, n$.

Thus, (3.7) becomes

$$
\left|h_{p}(x)-h_{p}(y)\right|<\varepsilon / 3+\left|\bar{h}_{\pi, p}(x)-\bar{h}_{\pi, p}(y)\right|+\varepsilon / 3,
$$

for all $y \in[0,1]$. We still need to find $\delta>0$ such that

$$
\left|\bar{h}_{\pi, p}(x)-\bar{h}_{\pi, p}(y)\right| \leq \varepsilon / 3 \text {, }
$$

provided $y \in(x-\delta, x+\delta)$. We first observe that if $\bar{f}_{\pi}$ and $\bar{g}_{\pi}$ are given by (3.8) and (3.9) respectively, then Lemma 7 implies that $\bar{h}_{\pi, p}$ must have the form

$$
\bar{h}_{\pi, p}=\sum_{i=1}^{n} c_{i} I_{\left(t_{i-1}, t_{i}\right]},
$$


for some real numbers $c_{1} \leq c_{2} \leq \cdots \leq c_{n}$. We now have only a few cases to consider.

Case 1. If $t_{j-1}<x<t_{j}$ for some $j \leq n$, then it follows that

$$
\left|\bar{h}_{\pi, p}(x)-\bar{h}_{\pi, p}(y)\right|=\left|c_{j}-c_{j}\right|=0<\varepsilon / 3,
$$

for all $y \in\left(t_{j-1}, t_{j}\right]$. Let $\delta=\min \left\{\left(x-t_{j-1}\right),\left(t_{j}-x\right)\right\}>0$ so (3.13) holds for all $y \in(x-\delta, x+\delta)$ and the continuity of $h_{p}$ at $x$ is established.

Case 2. $x=t_{j}$ for some $j<n$. Then (3.15) holds for all $y \in\left(t_{j-1}, x\right]$. Thus suppose $y \in\left(x, t_{j+1}\right]=\left(t_{j}, t_{j+1}\right]$, and suppose (3.13) does not hold, that is,

$$
\left|h_{\pi, p}(y)-h_{\pi, p}(x)\right|=h_{\pi, p}(y)-h_{\pi, p}(x)=c_{j+1}-c_{j}>\varepsilon / 3 .
$$

In Figure 1 below we fix $c_{j}, c_{j+1}$ and we may also without loss of generality fix $a_{j}, a_{j+1}$ and then we discuss briefly the various possibilities for the values of $\bar{b}_{j}, \bar{b}_{j+1}$ and each time we end up with a contradiction.

P1. If $a_{j+1}<\bar{b}_{j+1}<c_{j+1}$, then we may replace $c_{j+1}$ in (3.14) by $\max \left(c_{j}, \bar{b}_{j+1}\right)$ to obtain a better b.s.a. of $f$ and $g$, a contradiction. A similar conclusion holds if $c_{j}<\bar{b}_{j}<c_{j+1}$.

P2. If $\bar{b}_{j+1}<\bar{a}_{j+1}<c_{j+1}$, then we may replace $c_{j+1}$ by $\max \left(c_{j}, \bar{b}_{j+1}\right)$ to obtain a better b.s.a., a contradiction.

P3. If $\bar{b}_{j}>c_{j+1}>c_{j}$, then replace $c_{j}$ by $\bar{a}_{j}$ to obtain a better b.s.a, a contradiction. The same argument is valid if in addition we assume that $\bar{b}_{j+1}>c_{j+1}$.

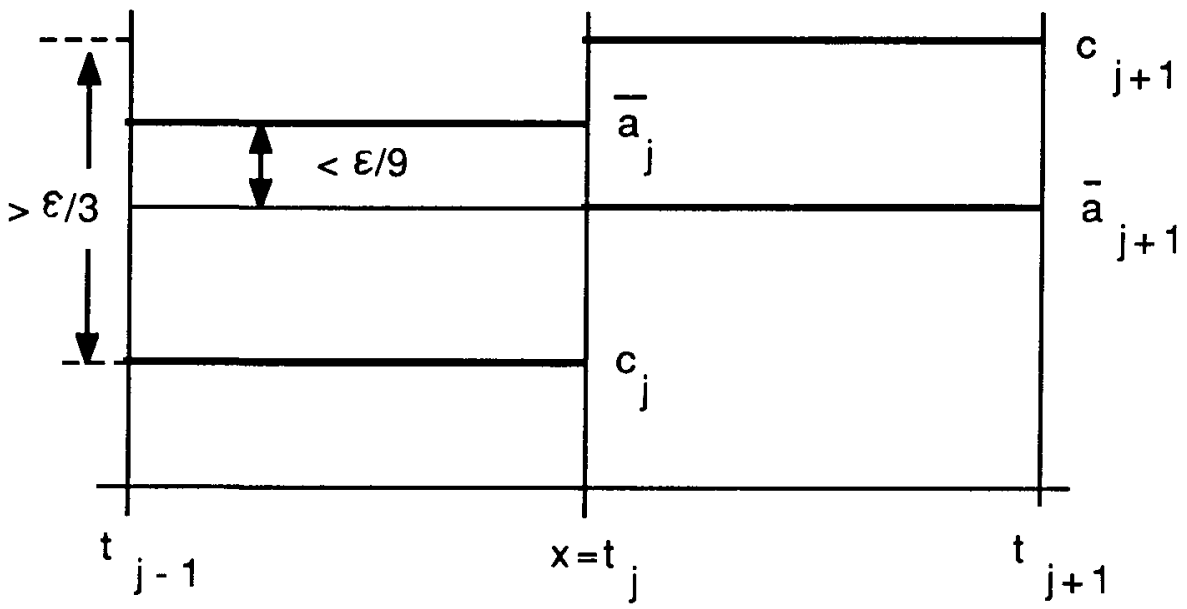

Figure 1 
P4. If $\bar{b}_{j}<c_{j}$ and $\bar{b}_{j+1}>c_{j+1}$, then $\bar{b}_{j+1}-\bar{b}_{j}>\varepsilon / 9$, contradicting (3.11).

\section{Examples}

EXAMPLE 1. Let $f$ be the real-valued step function defined on $[0,1]$ by (4.1)

$$
f=3 I_{[0,1 / 15]}+5 I_{(3 / 15,4 / 15]}+7 I_{(8 / 15,9 / 15]} \text {, }
$$

and let $g \equiv 0$ on $[0,1]$. Then $h_{2} \equiv 1 / 2$ on $[0,1]$ which is the same as the best $L_{2}$-approximant of the single function $(f+g) / 2$. This is always true, that is, the b.s.a. of $f$ and $g$ in the $L_{2}$-norm is equal to the best $L_{2}$-approximant of their mean [6, Theorem 3]. However, $h_{3}$ is not constant and it is given by

$$
h_{3}=(3 /(\sqrt{5}+1)) I_{[0,3 / 15]}+(5 / 4) I_{(3 / 15,8 / 15]}+(7 /(\sqrt{13}+1)) I_{(8 / 15,1]},
$$

while

$$
h_{\infty}=(3 / 2) I_{[0,3 / 15]}+(5 / 2) I_{(3 / 15,8 / 15]}+(7 / 2) I_{(8 / 15,1]} .
$$

In general if $g \equiv 0$ and $f$ is given by

$$
f=k_{1} I_{\left[0, t_{1}\right]}+k_{2} I_{\left(t_{2}, t_{3}\right]}+\cdots+k_{n} I_{\left(t_{2(n-1)}, t_{2 n-1}\right]},
$$

where

$$
2<k_{1}<k_{2}<\cdots<k_{n}
$$

and

$$
\begin{gathered}
t_{1}=\delta=\left(\sum_{j=1}^{n} k_{j}\right)^{-1}, \\
t_{2 i}=\left(\sum_{j=1}^{i} k_{j}\right) \delta, \quad j \geq 1, \\
t_{2 i+1}=t_{2 i} \delta, \quad i \geq 1,
\end{gathered}
$$

then for every $p, h_{p}$ must have the form

$$
h_{p}=\zeta_{1} I_{\left[0, t_{2}\right]}+\zeta_{2} I_{\left(t_{2}, t_{4}\right]}+\cdots+\zeta_{n} I_{\left(t_{2(n-1)}, 1\right]},
$$

where $0<\zeta_{1} \leq \zeta_{2} \leq \cdots \leq \zeta_{n}$ and $\zeta_{i}$ depends on $p$ for every $i$. Let us compute $h_{2}$ which has the form (4.4). Clearly $\zeta_{1}$ is the unique real number minimizing the quadratic function

$$
\begin{aligned}
T_{1}(\zeta) & =\delta\left(k_{1}-\zeta\right)^{2}+\delta(0-\zeta)^{2}+\left(t_{2}-t_{1}\right)(0-\zeta)^{2}+\left(t_{2}-t_{1}\right)(0-\zeta)^{2} \\
& =\delta\left(k_{1}-\zeta\right)^{2}+\delta \zeta^{2}+2 \delta\left(k_{1}-1\right) \zeta^{2}
\end{aligned}
$$


Differentiating $T_{1}$ we get

$$
\begin{aligned}
T_{1}^{\prime}\left(\zeta_{1}\right) & =-2 \delta\left(k_{1}-\zeta_{1}\right)+2 \delta \zeta_{1}+4 \delta\left(k_{1}-1\right) \zeta_{1} \\
& =2 \delta k_{1}\left(2 \zeta_{1}-1\right)=0 .
\end{aligned}
$$

Thus, $\zeta_{1}=1 / 2$. Similarly, $\zeta_{i}$ is the unique real number minimizing the function

$$
T_{i}(\zeta)=\delta\left(k_{i}-\zeta\right)^{2}+\delta \zeta^{2}+2 \delta\left(k_{i}-1\right) \zeta^{2}
$$

which implies that $\zeta_{i}=1 / 2$ for all $i \geq n$. Hence $h_{2} \equiv 1 / 2$ on $[0,1]$.

Suppose next, that we want to compute $h_{p}$ for $p>2$. Then in general $\zeta_{i}$ is the unique real number minimizing the function

$$
T_{i}(\zeta)=\delta\left(k_{i}-\zeta\right)^{p}+\delta \zeta^{p}+2 \delta\left(k_{i}-1\right) \zeta^{p} .
$$

Differentiating $T_{i}$, we get

$$
T_{1}^{\prime}\left(\zeta_{i}\right)=-p\left(k_{i}-\zeta_{i}\right)^{p-1}+p\left(2 k_{i}-1\right) \zeta_{i}^{p-1}=0,
$$

which implies upon dividing by $\left(p \zeta_{i}^{p-1}\right)$ that

$$
2 k_{i}-1=\left(\left(k_{i} / \zeta_{i}\right)-1\right)^{p-1}
$$

or

$$
\left(2 k_{i}-1\right)^{1 / \lambda}=\left(k_{i} / \zeta_{i}\right)-1, \quad \lambda=p-1,
$$

Hence

$$
\zeta_{i}=k_{i} /\left(\left(2 k_{i}-1\right)^{1 / \lambda}+1\right),
$$

for $i=1,2, \ldots, n$.

Now observe that $\zeta_{i} \rightarrow k_{i} / 2$ as $p=\lambda+1 \rightarrow \infty$ which implies that $h_{p}$ converges to a function

$$
h_{\infty}=\lim _{p \rightarrow \infty} h_{p}=\left(k_{1} / 2\right) I_{\left[t_{0}, t_{2}\right]}+\left(k_{2} / 2\right) I_{\left(t_{2}, t_{4}\right]}+\cdots+\left(k_{n} / 2\right) I_{\left(t_{2(n-1)}, 1\right]},
$$

which would be identical with the value of $h_{\infty}$ computed using (1.14).

It can also be shown that $\zeta_{i}$ increases as $k_{i}$ increases by differentiating (4.5) with respect to $k_{i}$ and observing that the derivative is always positive.

Notice that a function $h \in \mathscr{M}$ is a best simultaneous $L_{\infty}$-approximant of $f$ and $g$ if and only if $\underline{h} \leq h \leq \bar{h}$, where $\underline{h}, \bar{h} \in \mathscr{M}$ are given by $\bar{h} \equiv k_{n} / 2$ on $[0,1]$, and

$$
\underline{h}=\left(\left(2 k_{1}-k_{n}\right) / 2\right) I_{\left[t_{0}, t_{2}\right]}+\left(\left(2 k_{2}-k_{n}\right) / 2\right) I_{\left(t_{2}, t_{4}\right]}+\cdots+\left(k_{n} / 2\right) I_{\left(t_{2(n-1)}, 1\right]} \text {. }
$$

We show in Example 2 that the simultaneous Polya property does not hold in general for any two bounded Lebesgue measurable function $f$ and $g$, that is, the statement of Theorem 14(b) is not true in general. But before we proceed, we prove a little lemma. 
LEMMA 12. Let $f$ and $g$ be bounded Lebesgue measurable functions with $f \neq g$. Let $f_{p}$ and $g_{p}$ be the best $L_{p}$-approximant of $f$ and $g$ respectively by elements of $\mathscr{M}$, and let $h_{p}$ be their b.s.a. by elements of $\mathscr{M}$. Then

$$
\left\|f-g_{p}\right\|_{p} \geq\left\|f-h_{p}\right\|_{p},
$$

and

$$
\left\|g-f_{p}\right\|_{p} \geq\left\|g-h_{p}\right\|_{p} .
$$

Proof. By definition of $f_{p}$ and $g_{p}$ we have

$$
\left\|f-f_{p}\right\|_{p} \leq\left\|f-h_{p}\right\|_{p},
$$

and

$$
\left\|g-g_{p}\right\|_{p} \leq\left\|g-h_{p}\right\|_{p} .
$$

If (4.7) does not hold, then

$$
\left\|f-g_{p}\right\|_{p}<\left\|f-h_{p}\right\|_{p} .
$$

Combining this inequality with (4.10) implies that

$$
\left\|f-g_{p}\right\|_{p}^{p}+\left\|g-g_{p}\right\|_{p}^{p}<\left\|f-h_{p}\right\|_{p}^{p}+\left\|g-h_{p}\right\|_{p}^{p},
$$

or $g_{p}$ is a better b.s.a. of $f$ and $g$, a contradiction Therefore (4.7) must be true. Similarly we obtain (4.8). This completes the proof.

EXAMPLE 2. Next, let $f$ be the bounded Lebesgue measurable function given in [1] and defined on $[0,2]$. Let $g \equiv 7$ on $[0,2]$. Then clearly $g \in \mathscr{M}$ which implies that $g=g_{p} \equiv 7$ on $[0,2]$. Also $f$ as given is continuous on $[0,1) \cup(1,2]$ and approximately continuous at 1 . Let $\left\{p_{k}\right\}$ be as chosen in [1]. Then $7<f_{p_{2 n-1}}<7.5$ on [1,2] and $5<f_{p_{2 n}}<6$ on $[1,2]$, $n=1,2, \ldots$. Since $g_{p_{2 n-1}}=g_{p_{2 n}} \equiv 7$ on $[0,2]$. Then, we clearly have by the last lemma that $h_{p_{2 n-1}}>7$, otherwise we get $\left\|f-f_{p}\right\|_{p}>\left\|f-h_{p}\right\|_{p}$. Similarly for $p_{2 n}$ we must have $h_{p_{2 n}}<7$, otherwise we get $\left\|f-g_{p}\right\|_{p}<\left\|f-h_{p}\right\|_{p}$, a contradiction. Therefore the sequence $\left\{h_{p_{k}}\right\}_{k=1}^{\infty}$ does not converge anywhere.

\section{References}

[1] R. B. Darst and R. Huotari, 'Monotone approximation on an interval', Classical real analysis, edited by D. Waterman, pp. 43-44 (Contemp. Math. 42, Amer. Math. Soc. Providence, R.I., 1985).

[2] R. B. Darst and S. Sahab, 'Approximation of continuous and quasicontinuous functions by monotone functions', J. Approx. Theory 38 (1983), 9-27. 
[3] D. S. Goel, A. S. B. Holland, C. Nasim and B. N. Sahney, 'Characterization of an element of best $l^{p}$-simultaneous approximation', S. Ramanaujan Memorial Volume, Madras (1984), 10-14.

[4] A. S. B. Holland and B. N. Sahney, 'Some remarks on best simultaneous approximation', Theory of approximation with applications, edited by A. G. Law and B. N. Sahney, pp. 332-337 (Academic Press, New York, 1976).

[5] D. Landers and L. Rogge, 'On projections and monotony in $L_{p}$-spaces', Manuscripta Math 26 (1979), 363-369.

[6] G. M. Phillips and B. N. Sahney, 'Best simultaneous approximation in the $L_{1}$ and $L_{2}$ norms', Theory of approximation with applications, edited by A. G. Law and B. N. Sahney, pp. 332-337 (Academic Press, New York, 1976).

[7] V. A. Ubhaya, 'Isotone optimization II', J. Approx. Theory 12 (1974), 315-331.

[8] C. Van Eeden, Testing and estimating ordered parameters of probability distribution, (Ph.D. dissertation, University of Amsterdam, Studentendrukkerij Poortpers N.V., Amsterdam, 1958).

\section{King Abdulaziz University}

P.O. Box 9028

Jeddah 21413

Saudi Arabia 